\title{
ISCoS and WHO, an ever-strengthening cooperation for the benefit of individuals with spinal cord injuries worldwide
}

\author{
JJ Wyndaele \\ Antwerp University Hospital, Antwerp, Belgium \\ E-mail: spinalcord@uza.be
}

Dear Spinal Cord reader

In the year of the 50th anniversary of the International Spinal Cord Society (ISCoS), formerly known as the International Medical Society of Paraplegia (IMSoP), we are happy to report the further strengthening of the cooperation between ISCOS and the World Health Organization (WHO) for the benefit of individuals with spinal cord injury (SCI) worldwide.

IMSoP was founded in 1961 with Sir Ludwig Guttmann serving as the President. In February 1944, Dr Guttmann founded the Spinal Unit at Stoke Mandeville Hospital, Aylesbury, United Kingdom. In 1952 he also founded the International Stoke Mandeville Games, and health professionals from many countries who came with their teams to these Games began to meet and discuss their clinical work and research. It was decided to start IMSoP with its first official meeting to be held in Stoke Mandeville in 1961. Annual Scientific Meetings were held at Stoke Mandeville Hospital, except during Paralympic years when they were held in the country hosting the Games. IMSoP changed its name to ISCoS in 2001 to signal its broader objective, i.e. from paraplegia to all health aspects related to the spinal cord and to properly acknowledge the multiple aspects of comprehensive management, e.g. medical, nursing, physiotherapy, occupational therapy, psychology, engineering, research.

From 1979 onwards the ISCoS scientific meetings have been held in different countries, in order to support and encourage a global network as well as to better understand global perspectives of SCI issues. Meetings have been held in most continents, often jointly with local country or regional SCI organizations or in conjunction with the Paralympic Games. In addition, there are Regional Meetings around the world supported by the Society.

ISCoS serves as an international, non-political and non-profit-making association that aims to: study all potential topics relating to traumatic and non-traumatic SCI; work in close collaboration with other national and international organizations to encourage the most efficient use of available resources worldwide; contribute to scientific exchange by collecting and disseminating information through publications, correspondence, exhibits, regional and international seminars, symposia, conferences etc.; advise, encourage, guide and support the efforts of those responsible for the care of people with SCI; encourage research in the field of SCI by means of awards to authors with the best platform presentations and posters and young investigators publishing original papers in Spinal Cord; advise, encourage, guide and support and where appropriate facilitate the coordination of the education and training of medical professionals and professionals allied to medicine related to patients with SCI; and encourage the creation of affiliated societies worldwide. Many people have been instrumental in getting all these projects working and growing. They all deserve our deepest gratitude. To support membership from lowincome countries ISCoS keeps its membership fee very low, especially considering that it includes 12 issues of the journal Spinal Cord a year. In addition the society subsidizes both membership and attendance of Annual Scientific Meetings for professionals from the developing world.

In recent years, projects have been established between ISCoS and WHO to obtain results that can help in prevention, health promotion, treatment, rehabilitation and lifelong follow-up for individuals who sustain traumatic or develop a non-traumatic SCI.

Collaboration between ISCoS and WHO can be divided into several parts: (a) building capacity for better SCI education and prevention programmes; (b) improving classification systems for use of data in SCI research, the International Classification of External Cause of Injury (ICECI), the International Classification of Diseases (ICD), and the International Classification of Functioning, 
Disability and Health (ICF); and (c) improving the evidence base for SCI through the report, International Perspectives on Spinal Cord Injury (IPSCI). The objectives of the IPSCI report are first to summarize information on SCI, in particular the science and epidemiology, the services, interventions and policies that are relevant and 'the lived experience' of persons with SCI across the full spectrum of life and throughout the world; second, to document all aspects of the science and experience of SCI to identify gaps between what exists and what is required; and third to make recommendations based on this evidence, with a clear perception of feasible goals and targets, that are consistent with the aspirations and goals of inclusion and full participation as expressed in the UN Convention of the Rights of Persons with Disabilities.

ISCoS and WHO will continue to join forces in areas where they can make the greatest difference, especially in prevention and educational issues worldwide.

Spinal Cord (2011) 49, 677-678; doi:10.1038/sc.2011.61 